\title{
Phylogenetic Evidence that the Gram-Negative Nonsporulating Bacterium Tissierella (Bacteroides) praeacuta Is a Member of the Clostridium Subphylum of the Gram-Positive Bacteria and Description of Tissierella creatinini sp. nov.
}

\author{
J. A. E. FARROW, ${ }^{1}$ P. A. LAWSON,${ }^{1}$ H. HIPPE, ${ }^{2}$ U. GAUGLITZ, ${ }^{3}$ AND M. D. COLLINS ${ }^{1 *}$ \\ Institute of Food Research, Reading Laboratory, Reading RG6 2EF, United Kingdom, ${ }^{1}$ and Deutsche Sammlung \\ von Mikroorganismen und Zellkulturen GmbH, D-38124 Braunschweig, ${ }^{2}$ and BASF AG, \\ D-67056 Ludwigshafen, ${ }^{3}$ Germany
}

\begin{abstract}
The 16S rRNA gene sequence of the type strain of Tissierella praeacuta (formerly Bacteroides praeacutus) was determined by PCR direct sequencing. A comparative sequence analysis showed that $T$. praeacuta is a member of the Clostridium subphylum of the gram-positive bacteria and has a close phylogenetic affinity with the species that form Clostridium cluster XII (M. D. Collins, P. A. Lawson, A. Willems, J. J. Cordoba, J. Fernandez-Garayzabal, P. Garcia, J. Cai, H. Hippe, and J. A. E. Farrow, Int. J. Syst. Bacteriol. 44:812-826, 1994). Although T. praeacuta is gram negative and does not produce endospores, 16S rRNA sequence data showed that it is closely related genealogically (level of sequence similarity, 99.9\%) to Clostridium hastiforme. On the basis of our results and the results of previous studies, a second species of Tissierella, Tissierella creatinini sp. nov., is described.
\end{abstract}

Tissierella praeacuta (formerly Bacteroides praeacutus) consists of obligately anaerobic, gram-negative, nonsporulating, rod-shaped organisms and was originally isolated from infant feces and described by Tissier in 1908 (25). In addition to occurring in human feces, $T$. praeacuta has been isolated, although rarely, from a variety of clinical sources, including lung abscesses, gangrenous lesions, and blood $(1,2,11)$. Although well characterized, $T$. praeacuta has had an unsettled taxonomic history; at various times it has been assigned to the genera Coccobacillus (25), Zuberella (21), Fusobacterium (9), and Bacteroides (19). Shah and Collins (23), in a chemotaxonomic survey of the family Bacteroidaceae, showed that there were major differences between $B$. praeacutus and the group of species that belong to the genus Bacteroides sensu stricto (the "Bacillus fragilis group"). For example, B. praeacutus differs from true Bacteroides species by lacking menaquinones, by lacking several key metabolic enzymes, and by having a significantly lower DNA G+C content. Accordingly, Collins and Shah (4) transferred $B$. praeacutus to a new monospecific genus, the genus Tissierella. Although the genus Tissierella clearly differs from the genus Bacteroides and its relatives phenotypically, the higher phylogenetic associations of this taxon remain unknown.

There has been considerable progress in recent years in elucidating the phylogenetic relationships of the genus Bacteroides and other obligately anaerobic gram-negative bacteria by using $16 \mathrm{~S}$ rRNA sequence analysis (20). Therefore, in this study we determined the 16S rRNA gene sequence of the type strain of $T$. praeacuta in order to elucidate the higher phylogenetic affiliations of this organism. A comparative sequence analysis demonstrated that $T$. praeacuta is a member of the Clostridium subphylum of the gram-positive bacteria and is closely related genealogically to the type strain of Clostridium hastiforme. On the basis of the results of this study and previous studies a new creatinine-degrading species of the genus Tissierella, Tissierella creatinini, is described.

\footnotetext{
* Corresponding author. Phone: (734) 357226. Fax: (734) 267917.
}

\section{MATERIALS AND METHODS}

Bacterial strains. The type strains of $T$. praeacuta (ATCC 25539, NCTC 11158) and C. hastiforme (ATCC 33268, DSM 5675, NCTC 11832) were obtained from the American Type Culture Collection, Rockville, Md., the National Collection of Type Cultures, London, United Kingdom, and the Deutsche Sammlung von Mikroorganismen und Zellkulturen, Braunschweig, Germany, and were cultured as recommended in the culture collection catalogs.

Isolation of creatinine-degrading strain BN11 ${ }^{\mathrm{T}}$. Strain $\mathrm{BN} 11^{\mathrm{T}}(\mathrm{T}=$ type strain) was isolated from anaerobic enrichment cultures that had been inoculated with sediment from a wastewater pool at a sugar refinery in Nörten-Hardenberg, Germany. Enrichment medium AM0.1 contained (per liter) $1.17 \mathrm{~g}$ of $\mathrm{NaCl}, 0.4$ $\mathrm{g}$ of $\mathrm{MgCl}_{2} \cdot 6 \mathrm{H}_{2} \mathrm{O}, 0.3 \mathrm{~g}$ of $\mathrm{KCl}, 0.15 \mathrm{~g}$ of $\mathrm{CaCl}_{2} \cdot 2 \mathrm{H}_{2} \mathrm{O}, 0.27 \mathrm{~g}$ of $\mathrm{NH}_{4} \mathrm{Cl}, 0.2 \mathrm{~g}$ of $\mathrm{KH}_{2} \mathrm{PO}_{4}, 2.84 \mathrm{~g}$ of $\mathrm{Na}_{2} \mathrm{SO}_{4}, 1 \mathrm{ml}$ of trace element solution SL-10 (26), $1 \mathrm{ml}$ of

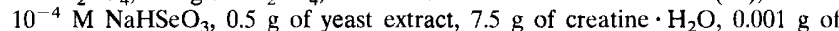
resazurin, $10 \mathrm{ml}$ of a vitamin solution $(28), 4.5 \mathrm{~g}$ of $\mathrm{NaHCO}_{3}$, and $0.5 \mathrm{~g}$ of L-cysteine hydrochloride; the $\mathrm{pH}$ of this medium was 7.5 . The medium was prepared anaerobically by the Hungate technique under an $80 \% \mathrm{~N}_{2}-20 \% \mathrm{CO}_{2}$ gas atmosphere. During autoclaving, $75 \%$ of the creatine was converted to creatinine (18). Enrichment cultures in completely filled 50-ml screw-cap bottles were incubated at $30^{\circ} \mathrm{C}$. After two transfers, a sample was streaked onto bicarbonate-free creatinine-containing AM0.1 agar plates and incubated in an anaerobic jar. Single colonies which produced a strong alkaline reaction when they were smeared on phenol red indicator paper were picked and purified by repeated streaking on agar plates of the same composition. Several isolates were obtained, and one strain, strain BN11 ${ }^{\mathrm{T}}$, was selected for further study.

Physiological and biochemical tests. Tests to determine production of lipase, lecithinase, urease, and indole, hemolysis, hydrolysis of gelatin, and digestion of milk and meat were performed as described by Holdeman et al. (10) and Krieg (14). Utilization of sugars and some other substances was determined in AM0.1 medium and in peptone-yeast extract medium at $\mathrm{pH} 6.8$ and 8.6. Utilization of creatinine and structurally related compounds was determined by using AM0.1 medium containing $0.5 \%$ yeast extract at $\mathrm{pH} 8.3$. Cultures were checked for spore production after growth in different liquid media or on agar media by phase-contrast microscopy and by performing heating tests. The $\mathrm{pH}$ and temperature optima and ranges for growth were determined in AM0.1 medium containing $1 \%$ yeast extract and $50 \mathrm{mM} \mathrm{N}$-methylhydantoin as the substrate. Different $\mathrm{pH}$ values were obtained by using only $1 \mathrm{~g}$ of sodium bicarbonate per liter and buffering the preparations with $0.02 \mathrm{M}$ potassium phosphate or Tris$\mathrm{HCl}$ buffer at $\mathrm{pH}$ values ranging from 5.8 to 9.5 . A model TN3 temperature gradient incubator (Tokyo Kagaku Sangyo, Ltd., Japan) was used to incubate cultures at temperatures ranging from 18 to $50^{\circ} \mathrm{C}$.

Analytical techniques. Acetate contents were determined by gas chromatography; creatinine and $N$-methylhydantoin contents were determined by highperformance liquid chromatography; sarcosine contents were determined enzymatically with sarcosine oxidase and peroxidase; and $N$-carbamoylsarcosine contents were determined with $N$-carbamoylsarcosine hydrolase in a coupled reaction with sarcosine oxidase as described previously (7). Ammonia levels were determined colorimetrically by the Berthelot reaction by using Merckognost urea (E. Merck, Darmstadt, Germany). 
DNA base composition. The $\mathrm{G}+\mathrm{C}$ content of strain $\mathrm{BN} 11^{\mathrm{T}}$ was determined by the thermal denaturation method with a Gilford model 2600 spectrophotometer equipped with a model 2527 thermoprogrammer and was calculated by using the equation of Marmur and Doty (17) as modified by De Ley (5). Escherichia coli K-12 strain DSM $485(\mathrm{G}+\mathrm{C}$ content, $51.7 \mathrm{~mol} \%)$ was used as the control.

16S rRNA gene sequence determination. Genomic DNA was extracted from mid-logarithmic-phase cells and was purified as described by Lawson et al. (15). 16S rRNA gene fragments were generated by a PCR, purified, and sequenced as described previously (12).

Analysis of sequence data. The sequence of $T$. praeacuta $\mathrm{NCTC} 11158^{\mathrm{T}}$ was aligned with 235 previously determined clostridial $(3,16)$ and reference sequences obtained from the EMBL and Ribosomal Database Project databases and was analyzed by using the DNADIST program of the PHYLIP package (6). The distance matrix which we obtained was corrected for substitution rates by using Kimura's parameters (13). A phylogenetic tree was constructed by the neighbor-joining method of Saitou and Nei (22) with a VAX computer. The stability of relationships was assessed by using the programs SEQBOOT, DNADIST, NEIGHBOR, and CONSENSE of the PHYLIP package. A total of 1,000 bootstrap trees were generated for the data set.

Nucleotide sequence accession numbers. The nucleotide sequences of the $16 \mathrm{~S}$ rRNAs of $T$. praeacuta NCTC $11158^{\mathrm{T}}$ and ATCC $25539^{\mathrm{T}}$ and $C$. hastiforme DSM $5675^{\mathrm{T}}$ have been deposited in the EMBL data library under accession numbers $\mathrm{X} 80832, \mathrm{X} 80833$, and $\mathrm{X} 80841$, respectively.

\section{RESULTS}

The 16S rRNA gene of $T$. praeacuta NCTC $11158^{\mathrm{T}}$ was amplified by a PCR, and its nucleotide sequence was determined directly. The sequence which we determined consisted of 1,475 nucleotides representing approximately $96 \%$ of the complete $16 \mathrm{~S}$ rRNA primary structure. This $16 \mathrm{~S}$ rRNA sequence was compared with the sequences of other 16S rRNAs available from the EMBL Data Library and the Ribosomal Database Project. Very low levels of sequence relatedness (generally $<77 \%$ ) were found with members of the genus Bacteroides and related gram-negative anaerobes. Much higher levels of sequence similarity were observed with members of the low-G+C-content subphylum of the gram-positive bacteria. Surprisingly, the $16 \mathrm{~S}$ rRNA sequence of $T$. praeacuta NCTC $11158^{\mathrm{T}}$ was found to be almost identical (level of similarity, $99.9 \%$, corresponding to 1 base difference in a comparison of 1,475 positions) to the sequence of the gram-positive spore-forming organism $C$. hastiforme DSM $5675^{\mathrm{T}}$. To eliminate the possibility of incorrect collection strains or culture contamination, cultures of the type strains of $T$. praeacuta (ATCC 25539) and $C$. hastiforme (ATCC 33268 and NCTC 11832) held in other collections were sequenced, and the original data were confirmed.

To determine the phylogenetic position of $T$. praeacuta within the Clostridium subphylum, the sequence of this organism was compared with previously published sequences of more than 200 clostridial species and non-spore-forming relatives of these organisms. A matrix of representative similarity values based on an approximately 1,330-nucleotide region of the 16S rRNA is shown in Table 1. In addition to its close genealogical affinity with $C$. hastiforme, $T$. praeacuta exhibited high levels of sequence relatedness with species belonging to Clostridium cluster XII (groups of Collins et al. [3]), which includes Clostridium acidiurici, Clostridium purinolyticum, "Clostridium filamentosum," Eubacterium angustum, and the previously unnamed non-spore-forming organism strain $\mathrm{BN} 11^{\mathrm{T}}$. A phylogenetic tree constructed by using the neighborjoining method is shown in Fig. 1, and this tree confirms the placement of $T$. praeacuta within this cluster.

\section{DISCUSSION}

The recovery of $T$. praeacuta within the Clostridium subphylum of gram-positive bacteria was somewhat unexpected as $T$. praeacuta stains gram negative and does not produce spores (4). However, there is a growing recognition that Gram stain- ing and spore formation are not necessarily good indicators of relatedness. For example, several genera that traditionally have been considered to be gram negative have now been shown to be related to gram-positive bacteria (e.g., members of the genera Pectinatus, Megasphaera, Selenomonas, and Sporomusa), while the phylogenetic intermixing of spore-forming and non-spore-forming taxa is now well established $(3,24,27)$. To our knowledge, however, this combination of differences in Gram staining and sporulation characteristics has not been reported previously in such phylogenetically closely related organisms. Although it is now recognized that the occurrence of identical (or nearly identical) 16S rRNA sequences does not necessarily imply that species are identical, the data demonstrate that $T$. praeacuta and $C$. hastiforme are either the same species or very closely related species. In the past $C$. hastiforme has been phenotypically associated with the group IV organisms Clostridium botulinum and Clostridium subterminale. Chromosomal DNA-DNA pairing studies are clearly necessary to determine whether $T$. praeacuta and $C$. hastiforme represent different species and the latter organism should be reclassified. The previously unnamed creatinine-degrading organism strain

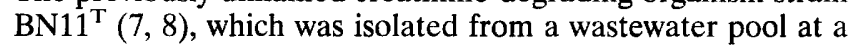
sugar refinery, exhibited $>95 \%$ sequence relatedness to $T$. praeacuta. Interestingly, strain $\mathrm{BN} 11^{\top}$ differs from $T$. praeacuta by being gram positive. Furthermore, unlike $T$. praeacuta, which has a wall based on meso-diaminopimelic acid, the wall of strain BN11 $1^{\mathrm{T}}$ contains D-ornithine as the dibasic amino acid. There are, however, now numerous examples of organisms which are genealogically closely related or members of the same genus but which differ in wall composition. Some examples within the Clostridium subphylum include the genus Clostridium sensu stricto (cluster I [3]), which contains species with meso-diaminopimelic acid and species with LL-diaminopimelic acid; and Clostridium paradoxum and Clostridium thermoalcalophilum, which exhibit approximately $2 \% 16 \mathrm{~S}$ rRNA sequence divergence and have walls based on meso-diaminopimelic acid and ornithine, respectively. Despite differences in staining and wall composition, $T$. praeacuta and strain $\mathrm{BN} 11^{\mathrm{T}}$ resemble each other in many ways, including the fact that they are both rod shaped and nonfermentative (carbohydrates are not utilized), the fact that they do not have endospores and respiratory menaquinones, and the fact that they have low DNA base compositions ( 28 to $32 \mathrm{~mol} \% \mathrm{G}+\mathrm{C}$ ). Thus, these similarities, together with the observed level of $16 \mathrm{~S}$ rRNA sequence divergence $(<5 \%)$, indicate that strain $\mathrm{BN} 11^{\mathrm{T}}$ should be included in the genus Tissierella. It is worth noting that grouping of strain $\mathrm{BN} 11^{\mathrm{T}}$ with $T$. praeacuta occurred in all 1,000 bootstrapped trees; these data support the close phylogenetic relationship of these organisms. Of the remaining species in Clostridium cluster XII, " $C$. filamentosum" exhibited approximately $10 \%$ sequence divergence with $T$. praeacuta and therefore in terms of evolutionary distance probably is peripheral to, or lies outside the boundaries of, the genus Tissierella. A relationship between " $C$. filamentosum" and the genus Tissierella was observed in only $83 \%$ of the bootstrapped trees. As pointed out previously (3), "C. filamentosum" could conceivably represent a distinct subline worthy of separate generic status. To avoid an undesirable proliferation of new genera, the genus Tissierella could be considered a possible genus of convenience for " $C$. filamentosum," although phenotypic information supporting this association would be required. $C$. acidiurici, C. purinolyticum, and E. angustum form a distinct group within cluster XII, exhibiting levels of sequence relatedness of $>93 \%$ with each other. Significantly lower levels of sequence similarity $(<90 \%)$ were observed with $T$. praeacuta and its relatives. The levels of sequence divergence and tree branching 
TABLE 1. Levels of sequence similarity for an approximately 1,330-nucleotide region of the 16S rRNAs of T. praeacuta and some representatives of the Clostridium subphylum of gram-positive bacteria

$\%$ Similarity to:

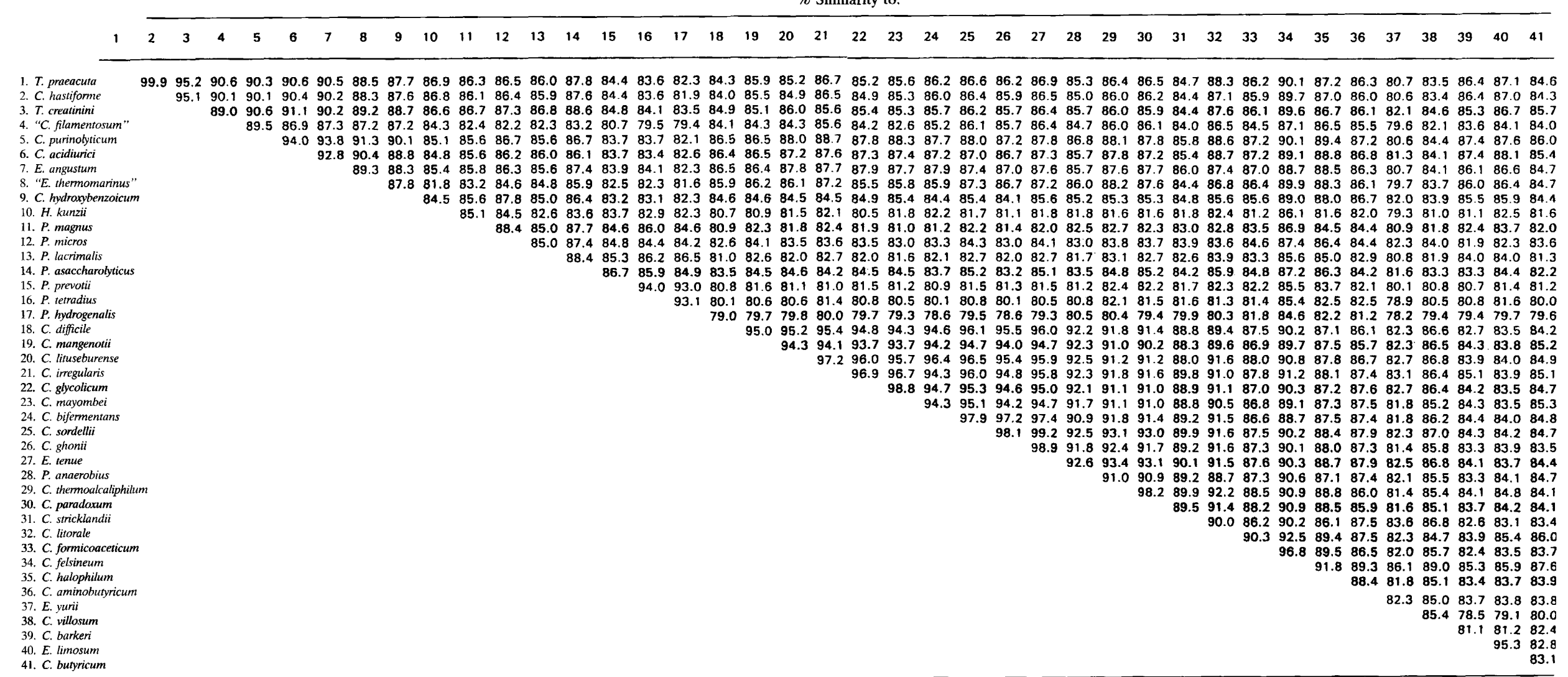

${ }^{a}$ Abbreviations: T., Tissierella; C., Clostridium; E., Eubacterium; H., Helcococcus; P., Peptostreptococcus. 


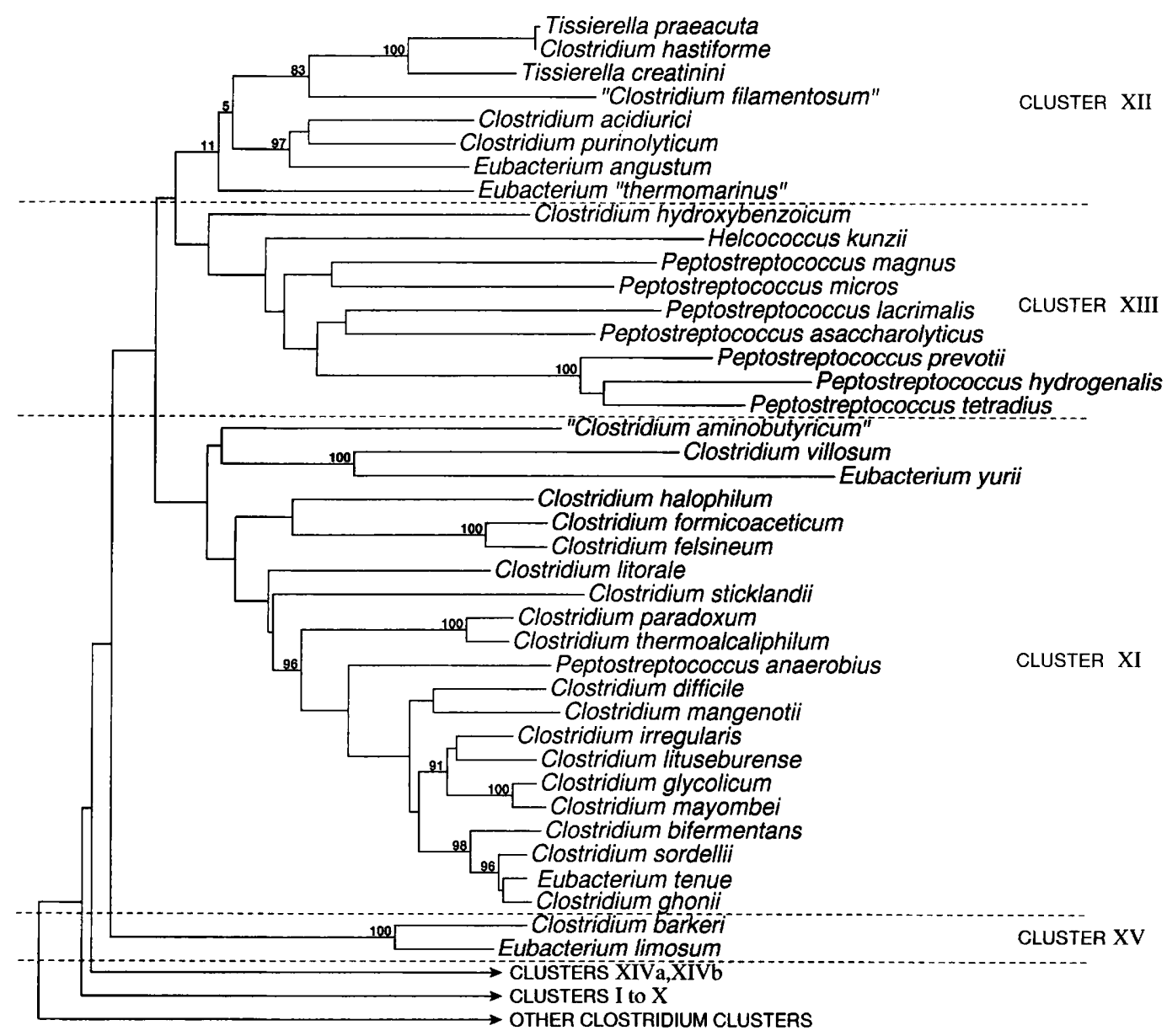

FIG. 1. Dendrogram showing the relationship of T. praeacuta to other members of the Clostridium subphylum of gram-positive bacteria. The tree was constructed by using the neighbor-joining method, and bootstrap values were calculated from 1,000 trees. Numbers are the percentages of bootstrap replicates.

considerations support the view that $C$. acidiurici, $C$. purinolyticum, and $E$. angustum represent a new genus that is related to, but nevertheless is separate from, the genus Tissierella.

Taxonomic proposals. (i) Emendation of the genus Tissierella (Collins and Shah). The description of the genus Tissierella given by Collins and Shah (4) should be emended as follows. Gram negative or gram positive: meso-Diaminopimelic acid or D-ornithine is present in the cell wall peptidoglycan. The cellular fatty acids are primarily straight-chain saturated and iso-methyl branched-chain or unsaturated fatty acids. Strains preferentially growing on creatinine typically produce acetate, sarcosine, $N$-carbamoylsarcosine, ammonia, and $\mathrm{CO}_{2}$. The $\mathrm{G}+\mathrm{C}$ content of the DNA is 28 to $32 \mathrm{~mol} \%$.

(ii) Description of Tissierella creatinini sp. nov. Tissierella creatinini (cre.a.ti.ni' ni. N. L. gen. n. creatinini, of creatinine). Cells are rod shaped (1.0 by $3.5 \mu \mathrm{m})$ and occur mostly singly or in pairs and rarely in chains of four to six cells (Fig. 2). Cells are nonmotile, and spores are not observed. Cells are gram positive and are surrounded by a slime capsule that is easily detectable in India ink preparations. Grows anaerobically in AM0.1 medium containing $0.05 \%$ yeast extract, $0.05 \%$ Trypticase, and creatinine or $N$-methylhydantoin. Growth is stimulated fivefold by the addition of formate $(40 \mathrm{mM})$, twofold by the addition of serine $(2 \mathrm{~g} /$ liter $)$ or threonine $(2 \mathrm{~g} / \mathrm{liter})$, and threefold by the addition of arginine ( $2 \mathrm{~g} /$ liter) and serine ( 2 $\mathrm{g} /$ liter), but not by the addition of arginine alone. In the $\mathrm{ab}-$ sence of creatinine or $N$-methylhydantoin, formate and amino acids do not support growth. No stimulation of growth is produced by lactate, malate, succinate, glycerol, or $\mathrm{H}_{2}$. Yeast extract (optimal concentration, 0.5 to $1.0 \%$ ) is required for growth and cannot be replaced by Casamino Acids or pep-

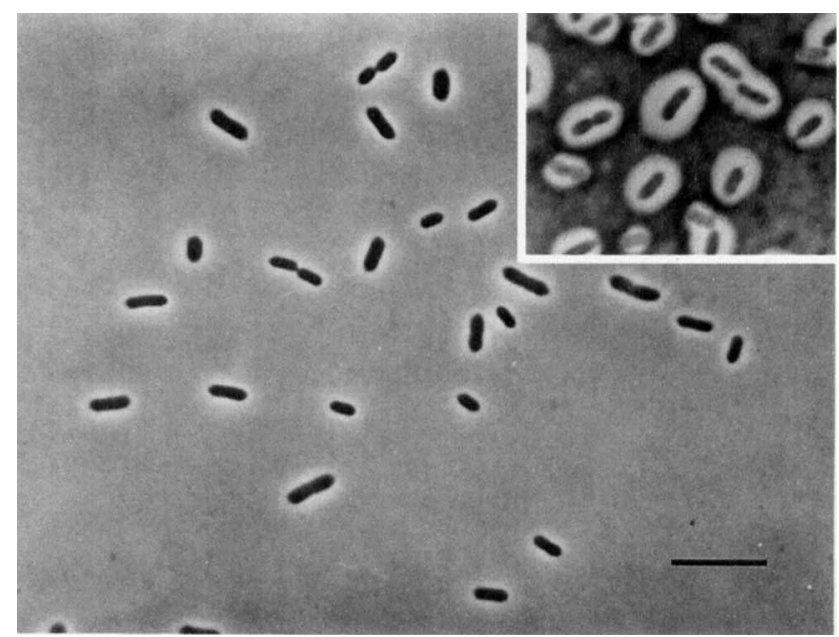

FIG. 2. Phase-contrast micrograph of strain $\mathrm{BN} 11^{\mathrm{T}}$. (Inset) India ink preparation showing slime capsules of cells. Bar $=10 \mu \mathrm{m}$. 
TABLE 2. Substrates utilized by $T$. creatinini $\mathrm{BN} 11^{\mathrm{T} a}$

\begin{tabular}{lcc}
\hline \multicolumn{1}{c}{ Substrate } & $\begin{array}{c}\text { Maximum optical } \\
\text { density at } 578 \mathrm{~nm}\end{array}$ & $\begin{array}{c}\text { Doubling time } \\
(\mathrm{h})\end{array}$ \\
\hline None & 0.170 & $\mathrm{ND}^{b}$ \\
Creatinine $(50 \mathrm{mM})$ & 1.740 & 4.2 \\
$N$-methylhydantoin $(50 \mathrm{mM})$ & 1.840 & 4.2 \\
$N$-carbamoylsarcosine $(50 \mathrm{mM})$ & 0.510 & 11.4 \\
Sarcosine $(50 \mathrm{mM})$ & 1.530 & 9.3 \\
Glycine $(50 \mathrm{mM})$ & 0.700 & 19.5 \\
Hydantoin $(50 \mathrm{mM})$ & 0.970 & 17.0 \\
Hydantoic acid $(50 \mathrm{mM})$ & 0.490 & 18.6 \\
\hline
\end{tabular}

${ }^{a}$ The medium used was AM0.1 medium containing $0.5 \%$ yeast extract. The optical densities of cultures grown in Hungate tubes were determined directly with a spectrophotometer (Bausch \& Lomb, Inc., Rochester, N.Y.). Doubling times were calculated from increases in optical density between 0.1 and 0.3 . The following substrates were tested but were not utilized: arabinose, cellobiose, fructose, glucose, inositol, lactose, maltose, mannitol, mannose, melibiose, raffinose, ribose, sucrose, salicin, sorbitol, trehalose, xylose, betaine, choline, methylamine, trimethylamine, lactate, pyruvate, glycerol, methanol, ethanol, formate, formamide, arginine, glutamate, serine, threonine, creatine, cytosine, and uracil.

${ }^{b} \mathrm{ND}$, not determined.

tones. Utilizes a limited range of mainly nitrogen-containing compounds, such as creatinine and structurally related substances, for growth. Not able to utilize creatine, carbohydrates, or various other substances (Table 2). Very weak growth occurs in peptone-yeast extract media (10), and biochemical tests for gelatin hydrolysis, production of lipase, lecithinase, indole, and urease, digestion of meat and milk, reduction of nitrate, and hemolysis are negative. Grows at 20 and $39^{\circ} \mathrm{C}$, and optimum growth occurs at $37^{\circ} \mathrm{C}$. The $\mathrm{pH}$ range for growth is 6.7 to 9.1, and the optimum $\mathrm{pH}$ is $\mathrm{pH} 8.3$ (determined with $N$-methylhydantoin as the substrate). Sodium bicarbonate and sodium chloride are required for good growth; the optimum concentrations of these compounds are 27 and $60 \mathrm{mM}$, respectively, in AM0.1 medium containing $0.5 \%$ yeast extract and $50 \mathrm{mM}$ $N$-methylhydantoin as the substrate. Creatinine is metabolized via $N$-methylhydantoin, $N$-carbamoylsarcosine, and sarcosine to acetate, ammonia, and $\mathrm{CO}_{2}$. In a typical growth experiment performed with AM0.1 medium containing $0.5 \%$ yeast extract, $2 \mathrm{~g}$ of serine per liter, $2 \mathrm{~g}$ of threonine per liter, and $46 \mathrm{mM}$ creatinine, the fermentation products formed after $60 \mathrm{~h}$ are 22 mmol of acetate per liter, $78 \mathrm{mmol}$ of ammonia per liter, 24 mmol of sarcosine per liter, and $7 \mathrm{mmol}$ of $N$-carbamoylsarcosine per liter. Respiratory menaquinones are not produced. The $\mathrm{G}+\mathrm{C}$ content of the DNA is $32 \mathrm{~mol} \%$. The cell wall murein is based on L-ornithine with D-glutamic acid as the interpeptide bridge (type $A 4 \beta$ ). Contains predominantly unsaturated fatty acids; $C_{18: 1 ~ c 11 / 12}(61 \%)$ is the major component. Minor amounts of straight-chain saturated, methylbranched, and 3-hydroxylated long-chain fatty acids are also produced. Isolated from a sugar refinery wastewater pond. The type strain is DSM 9508 (= BN11).

\section{ACKNOWLEDGMENTS}

This work was supported in part by a grant from the Ministry of Agriculture, Fisheries and Food.

We are grateful to R. M. Kroppenstedt and N. Weiss (Deutsche Sammlung von Mikroorganismen und Zellkulturen GmbH, Braunschweig, Germany) for chemotaxonomic characterization of strain $\mathrm{BN} 11^{\mathrm{T}}$.

\section{REFERENCES}

1. Beerens, H., and M. Demonchy. 1948. Sur une variété non gazogène de Zuberella praeacuta. C. R. Seances Soc. Biol. 142:1107-1108.
2. Cato, E. P., L. V. Holdeman, and W. E. C. Moore. 1979. Proposal of neotype strains for seven non-saccharolytic Bacteroides species. Int. J. Syst. Bacteriol. 29:427-434.

3. Collins, M. D., P. A. Lawson, A. Willems, J. J. Cordoba, J. FernandezGarayzabal, P. Garcia, J. Cai, H. Hippe, and J. A. E. Farrow. 1994. The phylogeny of the genus Clostridium: proposal of five new genera and eleven new species combinations. Int. J. Syst. Bacteriol. 44:812-826.

4. Collins, M. D., and H. N. Shah. 1986. Reclassification of Bacteroides praeacutus Tissier (Moore and Holdeman) in a new genus, Tissierella, as Tissierella praeacuta comb. nov. Int. J. Syst. Bacteriol. 36:461-463.

5. De Ley, J. 1970. Reexamination of the association between melting point buoyant density, and chemical base composition of nucleic acids. J. Bacteriol. 101:738-754.

6. Felsenstein, J. 1989. PHYLIP—phylogeny inference package (version 3.2). Cladistics 5:164-166.

7. Gauglitz, U. 1988. Anaerober mikrobieller Abbau von Kreatin, Kreatinin und N-Methylhydantoin. Reihe Biologie, Bd. 1. Unitext Verlag, Göttingen, Germany.

8. Gottschalk, E. M., H. Hippe, and F. Patzke. 1991. Creatinine deiminase (EC 3.5.4.21) from bacterium BNII: purification, properties and applicability in serum/urine creatinine assay. Clin. Chim. Acta 204:223-238.

9. Hoffman, H. 1957. Genus II. Fusobacterium Knorr, p. 436-440. In R. S. Breed, E. G. D. Murray, and N. R. Smith (ed.), Bergey's manual of determinative bacteriology, 7 th ed. The Williams \& Wilkins Co., Baltimore.

10. Holdemann, L. V., E. P. Cato, and W. E. C. Moore (ed.). 1977. Anaerobe laboratory manual, 4th ed. Virginia Polytechnic Institute and State University Anaerobe Laboratory, Blacksburg.

11. Holdeman, L. V., I. J. Good, and W. E. C. Moore. 1976. Human fecal flora: variation in bacterial composition within individuals and a possible effect of emotional stress. Appl. Environ. Microbiol. 31:359-375.

12. Hutson, R. A., D. E. Thompson, and M. D. Collins. 1993. Genetic interrelationships of saccharolytic Clostridium botulinum types B, E and F and related clostridia as revealed by small subunit rRNA sequences. FEMS Microbiol. Lett. 108:103-110.

13. Kimura, M. 1980. A simple method for estimating evolutionary rates of base substitutions through comparative studies of nucleotide sequences. J. Mol Evol. 16:111-120.

14. Krieg, N. R. 1981. Systematics, p. 407-449. In P. Gerhardt, R. G. E. Murray, R. N. Costilow, E. W. Nester, W. A. Wood, N. R. Krieg, and G. B. Phillips (ed.), Manual of methods for general bacteriology. American Society for Microbiology, Washington, D.C

15. Lawson, P. A., S. E. Gharbia, H. N. Shah, and D. R. Clark. 1989. Recognition of Fusobacterium nucleatum subgroups $\mathrm{Fn}-1, \mathrm{Fn}-2$ and $\mathrm{Fn}-3$ by ribosomal RNA gene restriction patterns. FEMS Microbiol. Lett. 65:41-46.

16. Lawson, P. A., P. L. Perez, R. A. Hutson, H. Hippe, and M. D. Collins. 1993. Towards a phylogeny of the clostridia based on 16S rRNA sequences. FEMS Microbiol. Lett. 113:87-92.

17. Marmur, J., and P. Doty. 1962. Determination of the base composition of DNA from its thermal denaturation temperature. J. Mol. Biol. 5:109-118.

18. Möller, B., H. Hippe, and G. Gottschalk. 1986. Degradation of various amine compounds by mesophilic clostridia. Arch. Microbiol. 145:85-90.

19. Moore, W. E. C., and L. V. Holdeman. 1973. New names and combinations in the genera Bacteroides Castellani and Chalmers, Fusobacterium Knorr, Eubacterium Prevot, Propionibacterium Orla-Jensen, and Lactobacillus Beijerinck. Int. J. Syst. Bacteriol. 23:69-74. (Author's correction, 24:311, 1974.)

20. Paster, B. J., F. E. Dewhirst, I. Olsen, and G. J. Fraser. 1994. Phylogeny of Bacteroides, Prevotella, and Porphyromonas spp. and related bacteria. J. Bacteriol. 176:725-732.

21. Prevot, A. R. 1938. Etudes de systematique bacterienne. Ann. Inst. Pasteur (Paris) 60:285-307.

22. Saitou, N., and M. Nei. 1987. The neighbor-joining method: a new method for reconstructing phylogenetic trees. Mol. Biol. Evol. 4:406-425.

23. Shah, H. N., and M. D. Collins. 1983. Genus Bacteroides: a chemotaxonomical perspective. J. Appl. Bacteriol 48:75-87.

24. Stackebrandt, E. 1992. Unifying phylogeny and phenotypic diversity, p. 1947. In A. Balows, H. G. Trüper, M. Dworkin, W. Harder, and K. H. Schleifer (ed.), The prokaryotes, vol. 1. Springer Verlag, New York.

25. Tissier, H. 1908. Recherche sur la flore intestinale normale des enfants ages d'un à cinq ans. Ann. Inst. Pasteur (Paris) 22:189-208.

26. Widdel, F., G. W. Kohring, and F. Mayer. 1983. Studies on dissimilatory sulfate-reducing bacteria that decompose fatty acids. III. Characterization of the filamentous gliding Desulfonema limicola gen. sp. nov. and Desulfonemo magnum sp. nov. Arch. Microbiol. 134:286-294.

27. Woese, C. R. 1987. Bacterial evolution. Microbiol. Rev. 51:221-271.

28. Wolin, E. A., R. S. Wolfe, and M. J. Wolin. 1964. Viologen dye inhibition of methane formation by Methanobacillus omelianskii. J. Bacteriol. 87:993-998. 